\title{
Area laws in quantum systems: mutual information and correlations
}

\author{
Michael M. Wolf ${ }^{1}$, Frank Verstraete ${ }^{2}$, Matthew B. Hastings ${ }^{3}$, J. Ignacio Cirac ${ }^{1}$ \\ ${ }^{1}$ Max-Planck-Institut für Quantenoptik, Hans-Kopfermann-Str.1, 85748 Garching, Germany. \\ ${ }^{2}$ Fakultät für Physik, Universität Wien, Boltzmanngasse 5, A-1090 Wien, Austria. \\ ${ }^{3}$ Center for Non-linear Studies and Theoretical Division, \\ Los Alamos National Laboratory, Los Alamos, New Mexico 87545, USA
}

(Dated: March 10, 2008)

\begin{abstract}
The holographic principle states that on a fundamental level the information content of a region should depend on its surface area rather than on its volume. In this paper we show that this phenomenon not only emerges in the search for new Planck-scale laws but also in lattice models of classical and quantum physics: the information contained in part of a system in thermal equilibrium obeys an area law. While the maximal information per unit area depends classically only on the number of degrees of freedom, it may diverge as the inverse temperature in quantum systems. It is shown that an area law is generally implied by a finite correlation length when measured in terms of the mutual information.
\end{abstract}

Correlations are information of one system about another. The study of correlations in equilibrium lattice models comes in two flavors. The more traditional approach is the investigation of the decay of two-point correlations with the distance. A lot of knowledge has been acquired in Condensed Matter Physics in this direction and is now being used and developed further in the study of entanglement in Quantum Information Theory [1, 2, 3]. The second approach (see Fig 1) asks how correlations between a connected region and its environment scale with the size of that region. This question has recently been addressed for a variety of quantum systems at zero temperature $[4,5,6,7,8,9,10,11,12,13$ ] where all correlations are due to entanglement which in turn is then measured by the entropy.

The original interest in this topic [12, 13, 14, 15] came from the insight that the entropy of black holes scales with the area of the surfaces at the event horizon-we say that an area law holds, in this case with a maximal information content of one bit per Planck area. Remarkably, a similar entropy scaling is observed in non-critical quantum lattice systems while critical systems are known to allow for small (logarithmic) deviations [6, 7, 8, 9, 10, 11]. Both is in sharp contrast to the behavior of the majority of states in Hilbert space which exhibit a volume scaling rather than an area law. These insights fruitfully guided recent constructions of powerful classes of ansatz states which are tailored to cover the relevant aspects of strongly correlated quantum many-body systems [16, 17].

A heuristic explanation of the area law in non-critical systems comes from the existence of a characteristic length scale, the correlation length, on which two-point correlations decay (Fig 1). Intuitively this apparent localization of correlations should imply an area law, an argument which can, however, not easily be made rigorous A firm connection between the decay of correlations and the area law is thus still lacking as well as is a proof and extension of the latter beyond zero temperature. In the present work we address both problems by resorting to a concept of Quantum Information Theory-the mutual information. The motivation for this quantity is that (i) it coincides with the entanglement entropy at zero temperature; (ii) it measures the total amount of information of one

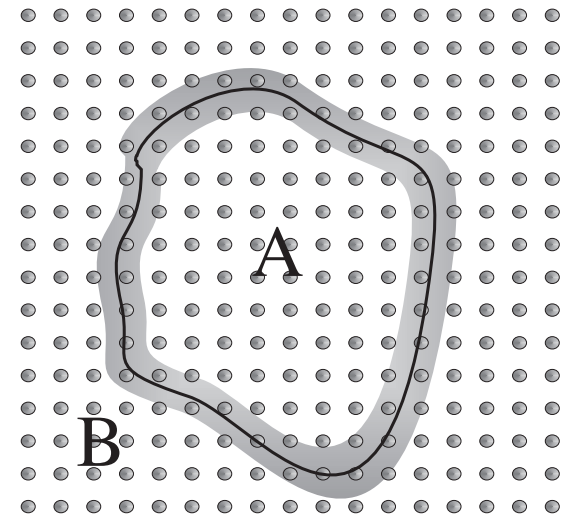

FIG. 1: We are interested in the mutual information (or entanglement) between the two regions $A$ and $B$. Heuristically, if there is a correlation length $\xi$ then sites in $A$ and $B$ that are separated by more than $\xi$ (the shaded stripe) should not contribute to the information or entanglement between $A$ and $B$. The mutual information (or entanglement) is thus bounded by the number of sites at the boundary.

system about another without 'overlooking' hidden correlations; (iii) the area law can be rigourously proven at any finite temperature; (iv) the heuristic picture relating decay of correlations and area law can be made rigorous in the form of a one-way implication. Moreover, we will prove that an area law is fulfilled by all mixed projected entangled pair states (PEPS), discuss the behavior of the mutual information for certain classes of 1D systems in more detail, and show that a strict 1D-area law implies that the state has an exact representation as a finitely correlated state.

We begin by fixing some notation. We consider systems on lattices $\Lambda \subseteq \mathbb{Z}^{\mathcal{D}}$ in $\mathcal{D}$ spatial dimensions which are sufficiently homogeneous (e.g., translational invariant). Each site of the lattice corresponds to a classical or quantum spin with configuration space $\mathbb{Z}_{d}$ or Hilbert space $\mathbb{C}^{d}$ respectively. Given a probability distribution $\rho$ on $\Lambda$ and marginals $\rho_{A}, \rho_{B}$ corresponding to disjoint sets $A, B \subseteq \Lambda$, the mutual information between these regions is defined by

$$
I(A: B)=H\left(\rho_{A}\right)+H\left(\rho_{B}\right)-H\left(\rho_{A B}\right),
$$


where $H(\rho)=-\sum_{x} \rho(x) \log \rho(x)$ is the Shannon entropy. In the quantum case the $\rho$ 's become density operators (and their partial traces) and $H$ has to be replaced by the von Neumann entropy $S(\rho)=-\operatorname{tr}[\rho \log \rho]$. The mutual information has a well defined operational meaning as the total amount of correlations between two systems [19]. It quantifies the information about $B$ which can be obtained from $A$ and vice versa. Elementary properties of the mutual information are positivity, that it vanishes iff the system factorizes, and it is non-increasing under discarding parts of the system [20]. We will occasionally write $S_{A}$ meaning $S\left(\rho_{A}\right)$.

Area laws for classical and quantum systems: Let us start considering classical Gibbs distributions of finite range interactions. All such distributions are Markov fields, i.e., if $x_{A}, x_{C}, x_{B}$ are configurations of three regions where $C$ separates $A$ from $B$ such that no interaction directly connects $A$ with $B$, then $\rho\left(x_{A} \mid x_{C}, x_{B}\right)=\rho\left(x_{A} \mid x_{C}\right)$ holds for all conditional probabilities [with $\rho(x \mid y):=\rho(x, y) / \rho(y)$ ]. Let us denote by $\partial A, \partial B$ the sets of sites in $A, B$ which are connected to the exterior by an interaction. Exploiting the Markov property together with the fact that we can express the mutual information in terms of a conditional entropy $H(A \mid B)=$ $H(A)-I(A: B)$ then leads to an area law

$$
I(A: B)=I(\partial A: \partial B) \leq H(\partial A) \leq|\partial A| \log d,
$$

where the first inequality follows from positivity of the classical conditional information. Equation (2) shows that correlations in classical thermal states are localized at the boundary. In particular if we take $B$ the complement of $A$, then we obtain that the mutual information scales as the boundary area of the considered region and the maximal information per unit area is determined by the number of microscopic degrees of freedom.

For quantum systems less information can be inferred from the boundary and the Markov property does no longer hold in general. Remarkably enough, for the case of the mutual information between a region $A$ and its complement $B$ we can also derive an area law for finite temperatures. In order to show that, we consider again a finite range Hamiltonian $H=H_{A}+H_{\partial}+H_{B}$, where $H_{A}, H_{B}$ are all interaction terms within the two regions and $H_{\partial}$ collects all those crossing the boundary. The thermal state $\rho_{A B}$ corresponding to the inverse temperature $\beta$ minimizes the free energy $F(\rho)=\operatorname{tr}[H \rho]-$ $\frac{1}{\beta} S(\rho)$. In particular, $F\left(\rho_{A B}\right) \leq F\left(\rho_{A} \otimes \rho_{B}\right)$ from which we obtain

$$
I(A: B) \leq \beta \operatorname{tr}\left[H_{\partial}\left(\rho_{A} \otimes \rho_{B}-\rho_{A B}\right)\right]
$$

since $H_{A}, H_{B}$ have the same expectation values in both cases. As the r.h.s. of Eq. (3) depends solely on the boundary we obtain again an area law scaling similar to that in Eq.(2). For example, if we just have two-site interactions we obtain $I(A: B) \leq 2 \beta\|h|\| \partial A|$, where $\| h\|\|$ is the maximal eigenvalue of all two-site Hamiltonians across the boundary, i.e., the strength of the interaction. Note that the scale at which the area law becomes apparent is now determined by the inverse temperature $\beta$. In fact, it is known that at zero temperature the boundary area scaling of the mutual information, which then becomes $I(A: B)=2 S(A)$, breaks down for certain critical systems [6, 7, 8, 9, 10, 11]. Eq.(3) shows that all the logarithmic corrections appearing in these models disappear at any finite temperature.

By comparing the area laws (2) and (3) we notice that quantum states may have higher mutual information than classical ones as the information per unit area is no longer bounded by the number of degrees of freedom. In fact, our results imply that if a system violates inequality (2), then it must have a quantum character. Note that Eqs. (23) directly generalize the findings of [21] for systems of harmonic oscillators.

Let us now turn to an important class of quantum states which goes beyond Gibbs states, namely projected entangled pair states (PEPS) [16]. These states bear their name from projecting 'virtual spins', obtained from assigning entangled pairs $|\Phi\rangle=\sum_{i=1}^{D}|i i\rangle$ to the edges of a lattice, onto physical sites corresponding to the vertices. A natural generalization of this concept to mixed states is to use completely positive maps for the mapping from the virtual to the physical level [18]. Since every such map can be purified, these mixed PEPS can be interpreted as pure PEPS with an additional physical system which gets traced out in the end. For all these states one can now easily see that the mutual information between a block $A$ and its complement $B$ satisfies a boundary area law

$$
I(A: B) \leq 2|\partial A| \log D,
$$

since it is upper bounded by the mutual information, i.e., twice the block entropy, of the purified state which is in turn bounded by the number of bonds cut. An interesting class of mixed PEPS are Gibbs states of Hamiltonians of commuting finite range interactions (see appendix). Note that these are not necessarily classical systems, as a simultaneous diagonalization need not preserve the local structure of the interaction. The Kitaev model [22] on the square lattice, the cluster state [23] Hamiltonian and all stabilizer Hamiltonians fall in this class. Moreover, Gibbs states of arbitrary local Hamiltonians are approximately representable as mixed PEPS [24].

Mutual information and correlations: We will now discuss the correlations measured in terms of the mutual information between separate regions. Traditionally, correlations are measured by connected correlation functions $\mathcal{C}\left(M_{A}, M_{B}\right):=$ $\left\langle M_{A} \otimes M_{B}\right\rangle-\left\langle M_{A}\right\rangle\left\langle M_{B}\right\rangle$ of observables $M_{A}, M_{B}$. In fact, these two concepts can be related by expressing the mutual information as a relative entropy $S\left(\rho_{A B} \mid \rho_{A} \otimes \rho_{B}\right)=I(A: B)$, using the norm bound $S(\rho \mid \sigma) \geq \frac{1}{2}\|\rho-\sigma\|_{1}^{2}[25]$ and the inequality $\|X\|_{1} \geq \operatorname{tr}[X Y] /\|Y\|$. In this way we obtain

$$
I(A: B) \geq \frac{\mathcal{C}\left(M_{A}, M_{B}\right)^{2}}{2\left\|M_{A}\right\|^{2}\left\|\mid M_{B}\right\|^{2}} .
$$

Hence, if $I(A: B)$ decays for instance exponentially in the distance between $A$ and $B$ then so will $\mathcal{C}$. One of the advantages of the mutual information is, that there cannot be correlations 'overlooked', whereas $\mathcal{C}$ might be arbitrarily small 

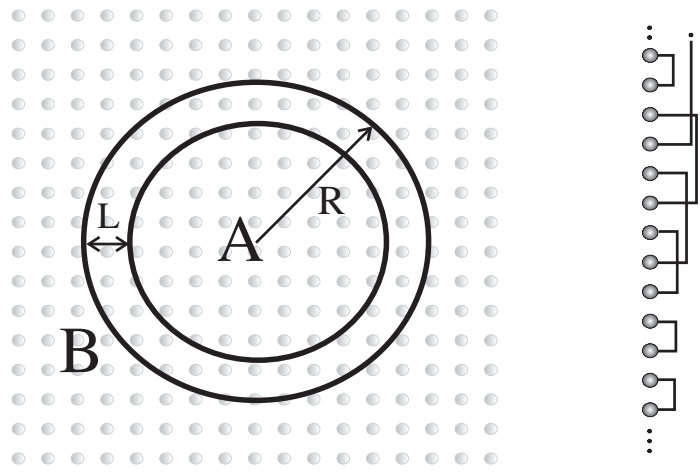

FIG. 2: Left: We consider regions $A$ and $B$ separated by a spherical shell of thickness $L \ll R$; Right: Simple 1D model for a state which is formed by singlet pairs (indicated by lines joining them) whose length follows a given probability distribution.

while the state is still highly correlated - a fact exploited in quantum data hiding and quantum expanders [26].

In the following we will relate the correlation length as defined by the mutual information with the area law mentioned previously. To this end consider a spherical shell $C$ of outer radius $R$ and thickness $L \ll R$ which separates the inner region $A$ from the exterior $B$ (see Fig. 2). We denote the mutual information between $A$ and $B$ by $I_{L}(R)$ and define $\xi_{M}$ as the minimal length $L$ such that $I_{L}(R)<I_{0}(R) / 2$ for all $R$, i.e., a correlation length measured by the mutual information. Note that $\xi_{M}$ can be infinite (e.g., for critical systems) and that it takes into account the decay of all possible correlations. Using the subadditivity property of the entropy we obtain the general inequality $I(A: B C) \leq I(A: B)+2 S_{C}$ which leads to

$$
I_{0} \leq I_{\xi_{M}}+2 S_{C} \leq 4|\partial A| \xi_{M} .
$$

Here the first inequality implies the second one by inserting $I_{\xi_{M}} \leq \frac{1}{2} I_{0}$ and the fact that $S(C) \leq \xi_{M}|\partial A|$. So, indeed, we get an area law for the mutual information solely from the existence of the length scale $\xi_{M}$, which expresses the common sense explanation of Fig. 1. This area law is also valid for zero temperature and when violated immediately implies an infinite correlation length $\xi_{M}$. The converse is, however, not true since there are critical lattice systems which obey an area law [21, 27]. Surprisingly, an area law can even hold under algebraically decaying two-point correlations [21, 27].

Examples in one dimension: We will now investigate the decay of correlations in terms of the mutual information for certain simple cases. We will show that in all of them $\xi_{M}$ is directly connected to the standard correlation length. We will consider infinite lattices in 1 spatial dimension (see Fig. 2).

We start out by considering an important class of states, the so-called finitely correlated states (FCS) [28], which naturally appear in several lattice systems in 1D. They can be viewed as 1D PEPS (or matrix product states). Every FCS is most easily characterized by a completely positive, trace preserving map (a channel) $T: \mathcal{B}\left(\mathcal{H}_{1}\right) \rightarrow \mathcal{B}\left(\mathcal{H}_{1} \otimes \mathcal{H}_{2}\right)$ with
$\mathcal{H}_{1}, \mathcal{H}_{2}$ Hilbert spaces of dimension $D, d$ respectively. Define further $\mathcal{E}(x)=\operatorname{tr}_{2}[T(x)]$ and assume the generic condition that $\mathcal{E}$ has only one eigenvalue of magnitude one. The second largest eigenvalue, $\eta$, is related to the standard correlation length through $\xi \sim-1 / \ln \eta$. In order to estimate $\xi_{M}$ we exploit the fact that $\rho_{A B}$ factorizes exponentially with increasing separation $L$, i.e., $\left\|\rho_{A B}-\rho_{A} \otimes \rho_{B}\right\|_{1}=\mathcal{O}\left(e^{-L / \xi}\right)$ (see appendix). Moreover, $T$ can be locally purified thereby increasing the size of $\mathcal{H}_{2}$ by a factor of $d D^{2}$. Denoting the additional purifying systems by $A^{\prime}$ and $B^{\prime}$ respectively, we obtain on the one hand $I(A: B) \leq I\left(A A^{\prime}: B B^{\prime}\right)=S\left(\rho_{A A^{\prime}} \otimes\right.$ $\left.\rho_{B B^{\prime}}\right)-S\left(\rho_{A A^{\prime} B B^{\prime}}\right)$. On the other hand we can apply Fannes' inequality [29], $|S(\rho)-S(\sigma)| \leq \Delta \log (\delta-1)+H(\Delta, 1-\Delta)$, where $\Delta=\frac{1}{2}\|\rho-\sigma\|_{1}$ and $\delta$ is the dimension of the supporting Hilbert space, to $I\left(A A^{\prime}: B B^{\prime}\right)$. Due to the purification we deal with finite dimensional systems $\left(\delta=D^{2}\right)$ so that putting things together leads to

$$
I_{L}(R) \leq \log (D) \mathcal{O}\left(L e^{-L / \xi}\right) .
$$

Since $I_{L}(R)$ increases (decreases) with $R(L)$, and is lower bounded by correlation function (5) this inequality immediately implies that $\xi_{M}$ is finite and directly related to $\xi$.

The case considered above includes several interesting situations of systems in 1D with finite-range interactions: frustration-free Hamiltonians at $T=0$, all classical Gibbs states, and all quantum ones for commuting Hamiltonians. In all cases, the area law is fulfilled following the results given in the previous sections. However, it is known that for certain critical local Hamiltonians the area law is violated at $T=0$. In order to analyze how this behavior may emerge, we will considered a simple toy model in $1 \mathrm{D}$ for which $I_{L}(R)$ can be exactly determined.

Let us consider a spin $\frac{1}{2}$ system formed of singlets (see Fig. 2). The state is such that from any given site, $i$, the probability of having a singlet with another site, $j$, is a function $f(|i-j|)$. The mutual information between two regions is equal to the number of singlets that connect those regions, and thus it can be easily determined (if we take a large region, so that we can average this number). If we take $f(x) \propto e^{-x / \xi}$ we have that: (i) all (averaged) correlation functions decay exponentially with the distance and that $\xi$ gives the correlation length; (ii) $I_{L}(R)$ decays exponentially with $L$ and that $\xi_{M} \sim \xi$; (iii) an area law is fulfilled. If we take $f(x) \propto 1 /\left(x^{2}+a^{2}\right)$ we obtain that: (i) the correlation functions decay as power laws with the distance; (ii) $I_{L}(R) \sim \log (2 R-L)$ and thus $\xi_{M}$ is infinite; (iii) the area law is violated. Thus, for this specific model we see how the violation of the area law naturally implies an infinite correlation length.

For zero temperature there is another simple connection between the area law and the decay of $I_{L}(R)$ as a function of the separation $L$. If for a pure state the entropy of a block of length $L$ goes to a constant $K$ as $S_{L}=K-f(L)$ with $f(L) \rightarrow 0$ for increasing $L$, then $I_{L}(R) \rightarrow f(L)$ as $R \rightarrow \infty$ for sufficiently large $L$. If the block entropy diverges instead, 
then $I_{L}(R) \rightarrow \infty$ for every finite separation.

Saturation of mutual information implies FCS: For onedimensional systems the area law just means a saturation of the mutual information. Let us finally gain some first insight into the structure of states having this property. So consider a general (mixed) 1D translational invariant state and denote the mutual information between a block of length $L$ and the rest of the system by $I(L)$ and similarly its entropy by $S(L)$. The latter can be shown to be a concave function

$$
S(L) \geq(S(L-1)+S(L+1)) / 2,
$$

which is nothing but the strong subadditivity inequality applied to a region of length $L-1$ surrounded by two single sites. Eq. (8) has strong implications on the behavior of $I(L)$. Assume that the system is a finite ring of length $N$, then

$$
\begin{aligned}
I(L)-I(L-1)= & {[S(L)-S(L-1)] } \\
& -[S(N-L+1)-S(N-L)]
\end{aligned}
$$

is a difference between two slopes of the entropy function. Due to concavity of $S(L), I(L)$ is increasing as long as $L<N / 2$. Moreover, if from some length scale on the mutual information exactly saturates, i.e., $I(L-1)=I(L)$ then all slopes between $L$ and $N-L$ have to be equal so that strong subadditivity in Eq.8 holds with equality. States with this property are, however, nicely characterized [30] and known to be quantum Markov chains. That is, there exists a channel $\tilde{T}: \mathcal{B}\left(\mathcal{H}_{2}^{\otimes(L-1)}\right) \rightarrow \mathcal{B}\left(\mathcal{H}_{2}^{\otimes L}\right)$ such that

$$
(\mathrm{id} \otimes \tilde{T})\left(\rho_{L-1}\right)=\rho_{L}
$$

where $\rho_{L}$ is the reduced density operator of $L$ sites and successive applications of $\tilde{T}$ to the last $L-1$ sites generates larger and larger parts of the chain. For infinite systems these states form a subset of the FCS where now $D=d^{(L-1)}$, i.e., the scale at which saturation sets in determines the ancillary dimension needed to represent the state.

Acknowledgements: Portions of this work were done at the ESI-Workshop on Lieb-Robinson Bounds. MBH was supported by US DOE DE-AC52-06NA25396. We acknowledge financial support by the European projects SCALA and CONQUEST and by the DFG (Forschungsgruppe 635 and Munich Center for Advanced Photonics (MAP)).

\section{APPENDIX}

In this appendix we show that (i) every Gibbs state of a local quantum Hamiltonian with mutually commuting interactions is a mixed projected entangled pair state (PEPS) with small bond dimension, and (ii) finitely correlated states factorize exponentially, i.e., exhibit an exponential split property.

\section{PEPS representation of thermal stabilizer states}

PEPS [16] bear their name from projecting 'virtual spins', obtained from assigning entangled pairs $|\Phi\rangle=\sum_{i=1}^{D}|i i\rangle$ to the edges of a lattice, onto physical sites corresponding to the vertices. A natural generalization of this concept to mixed states is to use completely positive maps for the mapping from the virtual to the physical level [18]. Since every such map can be purified, these mixed PEPS can be interpreted as pure PEPS with an additional physical system which gets traced out in the end. To become more specific let us consider a $2 \mathrm{D}$ square lattice. Then every pure PEPS is characterized by assigning a 5'th order tensor $A_{r, l, u, d}^{i}$ to each lattice site. Here the upper index corresponds to the physical site and the lower 'virtual' ones (running from 1 to $D$ ) get contracted according to the lattice structure. A mixed PEPS is then obtained by increasing the range of $i$ from $d$ to $d d_{E}$ and finally tracing over these additional environmental degrees of freedom, which can be thought of as a second layer of the square lattice.

Let us now prove that all Gibbs states of Hamiltonians of commuting finite range interactions are mixed PEPS. For simplicity consider again a $2 \mathrm{D}$ square lattice. Starting point is to write the un-normalized Gibbs state as $e^{-\beta H / 2} \mathbb{1} e^{-\beta H / 2}$ and to interpret the $\mathbb{1}$ as a partial trace over maximally entangled states $|\Phi \otimes \Phi\rangle$ to which $e^{-\beta H / 2}$ is applied. In order to get an explicit form for the tensor $A$ assume that horizontally neighboring sites interact via $h_{h}$ and vertical neighbors via $h_{v}$ and denote by

$$
e^{-\beta h_{v} / 2}=\sum_{\alpha} U_{\alpha} \otimes D_{\alpha}, \quad e^{-\eta h_{h} / 2}=\sum_{\beta} R_{\beta} \otimes L_{\beta}
$$

Schmidt decompositions in the Hilbert-Schmidt Hilbert space. That is, the operators $U_{\alpha}, D_{\alpha}, R_{\beta}, L_{\beta}$ form four sets of orthogonal operators, which by assumption commute with each other but not necessarily among themselves (e.g. $\left[U_{1}, U_{2}\right] \neq$ $0)$. Using that the Gibbs state is up to normalization a product of terms as in Eq.11) leads then to its PEPS representation with $D=d^{2}$ and

$$
A_{r, l, u, d}^{i}=\left[L_{r} R_{l} U_{d} D_{u}\right]_{i_{1}, i_{2}}
$$

where $i=\left(i_{1}, i_{2}\right)$ with $i_{2}$ corresponding to the environmental degrees.

\section{Decay of correlations for Finitely correlated states}

We consider now so called finitely correlated states (FCS) [28], which naturally appear in several lattice systems in 1D. They can be viewed as 1D PEPS (or matrix product states) where all the local projectors are the same. Every FCS is most easily characterized by a completely positive, trace preserving map (a channel) $T: \mathcal{B}\left(\mathcal{H}_{1}\right) \rightarrow \mathcal{B}\left(\mathcal{H}_{1} \otimes \mathcal{H}_{2}\right)$ with $\mathcal{H}_{1}, \mathcal{H}_{2}$ Hilbert spaces of dimension $D, d$ respectively. Define further $\mathcal{E}(x)=\operatorname{tr}_{2}[T(x)]$ and assume the generic condition that $\mathcal{E}$ has only one eigenvalue of magnitude one, corresponding to a 
fixed point $\varrho=\mathcal{E}(\varrho)$. The second largest eigenvalue, $\eta$, is related to the standard correlation length through $\xi=-1 / \ln \eta$. With this notation, it is very simple to express the states corresponding to regions $A, B$, and $A B$ (which are required in order to determine the mutual information). We now show that as $L$ gets larger, $\rho_{A B}$ approaches exponentially fast $\rho_{A} \otimes \rho_{B}$.

The reduced density matrix $\rho_{A}$ of $N_{A}=R-L$ contiguous sites is obtained as

$$
\rho_{A}=\operatorname{tr}_{1}\left[T^{N_{A}}(\varrho)\right]
$$

Similarly the joint reduced state of two regions $A$ and $B$ which are separated by $L$ sites is given by

$$
\rho_{A B}=\lim _{N_{B} \rightarrow \infty} \operatorname{tr}_{1}\left[T^{N_{B}} \mathcal{E}^{L} T^{N_{A}} \mathcal{E}^{L} T^{N_{B}}(\varrho)\right] .
$$

For sufficiently large $L$ write

$$
\mathcal{E}^{L}(x)=\left(1-c \eta^{L}\right) \operatorname{tr}[x] \varrho+c \eta^{L} \mathcal{E}^{\prime}(x),
$$

where $\mathcal{E}^{\prime}$ is some channel and $c$ an $L$-independent constant. Taken together Eqs. 13 15 enable us to bound the norm distance

$$
\left\|\rho_{A B}-\rho_{A} \otimes \rho_{B}\right\|_{1} \leq 4 c \eta^{L}
$$

independent of $N_{A}, N_{B}$. That is, the two regions factorize exponentially on a scale $\xi=-1 / \ln \eta$ which can be regarded the correlation length of the system. We cannot use this result directly for the mutual information since the dimension of the Hilbert space of system $B$ is infinite. However, we can proceed by noting that each $T$ can be locally purified thereby increasing the size of $\mathcal{H}_{2}$ by a factor of $d D^{2}$ (with $\mathcal{E}$ unchanged). Denoting the additional purifying systems by $A^{\prime}$ and $B^{\prime}$ respectively, we obtain on the one hand $I(A: B) \leq$ $I\left(A A^{\prime}: B B^{\prime}\right)=S\left(\rho_{A A^{\prime}} \otimes \rho_{B B^{\prime}}\right)-S\left(\rho_{A A^{\prime} B B^{\prime}}\right)$. On the other hand we can apply Fannes' inequality, $|S(\rho)-S(\sigma)| \leq$ $\Delta \log (\delta-1)+H(\Delta, 1-\Delta)$, where $\Delta=\frac{1}{2}\|\rho-\sigma\|_{1}$ and $\delta$ is the dimension of the supporting Hilbert space, to $I\left(A A^{\prime}: B B^{\prime}\right)$. The advantage is that in this system we deal with finite dimensional systems with $\delta=D^{2}$.

[1] A. Osterloh, L. Amico, G. Falci, and R. Fazio, Nature 416, 608 (2002).

[2] T.J. Osborne and M.A. Nielsen, Phys. Rev. A 66, 032110 (2002).

[3] F. Verstraete, M. Popp, and J. I. Cirac, Phys. Rev. Lett. 92, 027901 (2004).

[4] M. Plenio, J. Eisert, J. Dreißig, and M. Cramer, Phys. Rev. Lett. 94, 060503 (2005).

[5] A. Botero and B. Reznik, Phys. Rev. A 70, 052329 (2004).

[6] H. Casini, C.D. Fosco, and M. Huerta, J. of Stat. Mech. 507, 007 (2005).

[7] G. Vidal, J.I. Latorre, E. Rico, A. Kitaev, Phys. Rev. Lett. 90, 227902 (2003)

[8] B.-Q- Jin and V. Korepin, J. Stat. Phys. 116, 79 (2004).
[9] P. Calabrese, J. Cardy, J. Stat. Mech. P06002 (2004).

[10] M.M. Wolf, Phys. Rev. Lett. 96, 010404 (2006).

[11] D. Gioev, I. Klich, Phys. Rev. Lett. 96, 100503 (2006).

[12] L. Bombelli, R.K. Koul, J. H. Lee, and R. D. Sorkin, Phys. Rev. D 34, 373 (1986).

[13] M. Sredniicki, Phys. Rev. Lett. 71, 666 (1993).

[14] G.'t Hooft, Nucl. Phys. B 256, 727 (1985).

[15] C.G. Callan and F. Wilczek, Phys. Lett. B 333, 55 (1994).

[16] F. Verstraete, J.I. Cirac. Renormalization algorithms for Quantum-Many Body Systems in two and higher dimensions. http:/xxx.lanl.gov/cond-mat/0407066 (2004).

[17] G. Vidal, cond-mat/0512165

[18] F. Verstraete, J.J. Garcia-Ripoll, J.I. Cirac, Phys. Rev. Lett. 93, 207204 (2004).

[19] B. Groisman, S. Popescu, and A. Winter, Phys. Rev. A 72, 032317 (2005).

[20] M. Nielsen and I. Chuang, Quantum computation and quantum information, Cambridge University Press, 2000.

[21] M. Cramer, J. Eisert, M.B. Plenio, and J. Dreißig, Phys. Rev. A 73, 012309 (2006).

[22] A.Y. Kitaev, Annals of Physics 303, 2 (2003).

[23] R. Raussendorf, H.J. Briegel, Phys. Rev. Lett. 86, 910 (2001).

[24] M. B. Hastings, Phys. Rev. B 73, 085115 (2006).

[25] M. Ohya, D. Petz, Quantum Entropy and its Use, Springer, Heidelberg (1993).

[26] P. Hayden, D. Leung. P.W. Shor, and A. Winter, Comm. in Math. Phys. 250 371 (2004); M. B. Hastings, cond-mat/0701055 (2007); Ben-Aroya and Ta-Shma, quant-ph/0702129 (2007).

[27] F. Verstraete, M.M. Wolf, D. Perez-Garcia, J.I. Cirac, Phys. Rev. Lett. 96, 220601 (2006).

[28] M. Fannes, B. Nachtergaele, R.F. Werner, Commun. Math. Phys. 144, 443 (1992).

[29] K.M.R. Audenaert, J. Phys. A 40, 8127 (2007).

[30] P. Hayden, R. Jozsa, D. Petz, A. Winter, Commun. Math. Phys. 246, 359 (2004). 